\title{
Methods for scalable optical quantum computation
}

\author{
Tal Mor ${ }^{1}$ and Nadav Yoran ${ }^{1,2}$ \\ ${ }^{1}$ Computer Science Department, Technion, Haifa 32000, Israel \\ ${ }^{2}$ H.H.Wills Physics Laboratory, University of Bristol, Tyndall Avenue, Bristol BS8 1TL, UK
}

(Dated: August 28, 2018)

\begin{abstract}
We propose a scalable method for implementing linear optics quantum computation using the "linked-state" approach. Our method avoids the two-dimensional spread of errors occurring in the preparation of the linked-state. Consequently, a proof is given for the scalability of this modified linked-state model, and an exact expression for the efficiency of the method is obtained. Moreover, a considerable improvement in the efficiency, relative to the original linked-state method, is achieved. The proposed method is applicable to Nielsen's optical "cluster-state" approach as well.
\end{abstract}

Although very successful in implementing various protocols of quantum information, optical approaches were not considered to be a useful tool for implementing scalable quantum computation. The reason is the lack of interaction between photons required for the implementation of two-qubit gates. It was, however, shown by Knill, Laflamme and Milburn (KLM) [1] that this obstacle can be overcome. KLM have shown that a probabilistic two-qubit gate can be implemented by means of interference with ancillary photons and incomplete measurements (detecting only a part of the photons). The resulting conclusive measurement or unambiguous detection sometimes yields the correct quantum output, and the user can identify these instances as these are accompanied by a specific classical outcome of the measurement. The probability of success of any KLM-type gate is determined by the complexity of the ancillary state. The more resources, in terms of the number of elementary operations, invested in the preparation of the ancilla, the higher the success probability. The basic requirements of the KLM scheme are single-photon sources, photon counters (that distinguish between different number states) and fast feed-forward ability. It was shown by KLM that by using their gates scalable quantum computation can be implemented. Yet, a huge resource overhead is required in order to reduce the unavoidable probability to fail in each gate application. Additionally, scalability is achieved (even in the ideal case where all optical devices are error-free) only if error correcting codes are employed.

The problem of the unavoidable possibility of failing occurring in each KLM-type gate (which leads to the enormous resource overhead) was recently solved by a new scheme 2]. The information processing in this scheme is done in two stages, which we call here "construction" and "evolution". In the construction stage a multi-photon "linked-state" is prepared, corresponding to the specific computation. The subsequent evolution stage is then fully deterministic. The linked-state consists of chains of photons, each chain corresponding to a single logical qubit. In such a chain the path degree of freedom of each photon is maximally entangled with the polarization of the next photon. The construc- tion stage includes the preparation of the above chains and the "weaving" of the chains together into the overall linked-state. The weaving steps are done by entangling photons of different chains according to the circuit one wishes to process. Both the chain-preparation and the weaving are done in parallel using KLM-type gates via unambiguous detection. In the evolution stage the data, which is initially encoded in the polarization of the first photon in the chain, progresses along the chain (from the polarization of one photon to that of the next photon) by a sequence of teleportation steps. In each such teleportation step both (path and polarization) degrees of freedom of a single photon are measured in the Bell basis; a single teleportation of this type was experimentally demonstrated in [3]. One-qubit gates are implemented by rotating the polarization of the photon that carries the data prior to teleporting it. A conditional phase gate (CPHASE) is induced on two logical qubits in any event where the data in the two corresponding chains passes through two photons that were entangled by a weaving step.

The linked-state can therefore be viewed as a two dimensional structure consisting of connected chains. This method exhibits vastly improved efficiency over the KLM method (see [2]), and furthermore, it is believed that this scheme is scalable even without the need for inherent error correction. Yet, so far, due to the complex dynamics of the construction process there is no proof for the scalability of the linked-state scheme, let alone a calculation of its efficiency. When a KLM-type gate fails and a photon is removed, the attempt to replace it by a new photon may also fail, resulting in the removal of one of the photons that were connected to it as well. A failure can, therefore, spread in this manner both backwards in one chain and, worse, also to other chains. Efficiency results appearing in 2] were based on simulations of a simple circuit where two-qubit gates are repeatedly applied to just a pair of logical qubits. A full proof of scalability requires the analysis of a rather complex 2-dimensional random walk process, and was not done yet.

A different yet somewhat similar approach, suggested by Nielsen [4], is the optical version of the cluster-state 
model of Raussendorf and Briegel [5] (further developments are described in [6]). This method also requires the preparation of a multi-photon 2-dimensional structure of inter-connected chains. It should be noted that a previous estimation [4] of the efficiency of his method does not take into account all (two-dimesnional) paths through which a gate failure can spread - measured photons that have more than a single connection to the rest of the cluster are more expansive to replace.

Here we present a scalable linked-state method and prove its scalability. The key feature of the method is that it avoids the two-dimensional spread of errors. Therefore, it avoids the need for a complex 2-dimensional random walk analysis. In our method, failures in probabilistic gates that might spread backwards are confined to the chain preparation steps. The connections between the chains are established in a manner that prevents any spread of failures during the weaving steps. Consequently, we are able to provide an exact expression for the efficiency of the method. In addition, the new linkedstate method is considerably more efficient then the original one. The proposed method can be applied, with the same advantages, to design a scalable optical cluster-state method as well.

The basic idea is to construct for each logical qubit a somewhat more complex chain of photons (than in [2]). The polarization of each such "linked-photon" in such a chain is entangled not only with the path degree of freedom of the preceding linked-photon in the chain, but also with the path of an additional photon, a "free-arm" photon. A schematic description of the chain is given in Fig. 1. Only the free-arm photons are then used in the weaving steps to establish a connection between two chains. This is done by applying to these photons a probabilistic KLM-type gate. A successful application of the gate will produce the entangled state required for the application of a two-qubit CPHASE gate as in the original linked-state method. A failure of a KLM-type gate results in the measurement of (exactly) one of its input photons. Such a failure in the connecting operation will therefore result in the measurement of one of the freearm photons. However, no other part of that chain will be affected. Therefore the transmission of the data along the chain is still possible, and a second attempt to establish a connection can be applied to the next free-arm photon in the chain, and so on. The state of such a chain is the following

$$
\begin{gathered}
|\uparrow\rangle_{p_{1}}\left(|0\rangle_{p_{1}}|\uparrow\rangle_{p_{2}}|+\rangle_{p_{2^{\prime}}}+|1\rangle_{p_{1}}|\leftrightarrow\rangle_{p_{2}}|-\rangle_{p_{2^{\prime}}}\right) \\
\left(|0\rangle_{p_{2}}|\uparrow\rangle_{p_{3}}|+\rangle_{p_{3^{\prime}}}+|1\rangle_{p_{2}}|\leftrightarrow\rangle_{p_{3}}|-\rangle_{p_{3^{\prime}}}\right) \cdots \\
\left(|0\rangle_{p_{n-1}}|\uparrow\rangle_{p_{n}}|+\rangle_{p_{n^{\prime}}}+|1\rangle_{p_{n-1}}|\leftrightarrow\rangle_{p_{n}}|-\rangle_{p_{n^{\prime}}}\right)|0\rangle_{p_{n}}
\end{gathered}
$$

where $\{|0\rangle,|1\rangle\}$ and $\{|\uparrow\rangle,|\leftrightarrow\rangle\}$ denote the path and

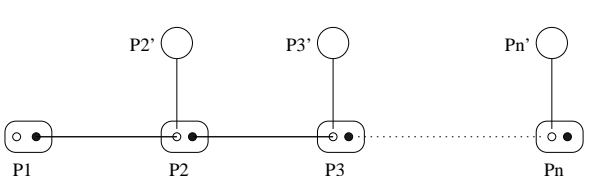

FIG. 1: A chain state for our linked state model. The rectangles represent the linked-photons whose polarization and path degree of freedom are denoted by empty and full circles respectively. The connected circles (denoted with primes) signify the free-arm photons of which only the path degree of freedom is utilized.

polarization of each photon respectively; and $|+\rangle=(|0\rangle+$ $|1\rangle) / \sqrt{2}$ and $|-\rangle=(|0\rangle-|1\rangle) / \sqrt{2}$ are the states of the photons that constitute the free-arms (denoted by primed indices). The terms within the brackets are the links of the chain, carried by three physical degrees of freedom (of three different photons) in a GHZ state.

Let us now discuss in detail a single weaving step connecting two chains using the free-arm photons. The initial states of the links that are to be connected are given by: $\left(|0\rangle_{p_{1}}|\uparrow\rangle_{p_{2}}|+\rangle_{p_{2^{\prime}}}+|1\rangle_{p_{1}}|\leftrightarrow\rangle_{p_{2}}|-\rangle_{p_{2^{\prime}}}\right)$ and $\left(|0\rangle_{q_{1}}|\uparrow\rangle_{q_{2}}|+\rangle_{q_{2^{\prime}}}+|1\rangle_{q_{1}}|\leftrightarrow\rangle_{q_{2}}|-\rangle_{q_{2^{\prime}}}\right)$. The desired final state - after measuring $p^{\prime}$ and $q^{\prime}$ - is the following:

$$
\begin{aligned}
& \left|0_{p_{1}} \uparrow_{p_{2}}\right\rangle\left(\left|0_{q_{1}} \uparrow_{q_{2}}\right\rangle+\left|1_{q_{1}} \leftrightarrow_{q_{2}}\right\rangle\right)+ \\
& \left|1_{p_{1}} \leftrightarrow_{p_{2}}\right\rangle\left(\left|0_{q_{1}} \uparrow_{q_{2}}\right\rangle-\left|1_{q_{1}} \leftrightarrow_{q_{2}}\right\rangle\right)
\end{aligned}
$$

This entangled state of four degrees of freedom of four different photons is the basic unit which is required for the application of a two-qubit gate in the linked-state model [2]. This entanglement between the links is established by applying a KLM-type CPHASE gate to $p_{2^{\prime}}$ and $q_{2^{\prime}}$. This gate [1] consists of two teleportation protocols based on the Fourier transform $\left(\hat{F}_{n+1}\right)$. In addition to one input photon, $n$ ancillary photons take part in each of these " $F$-teleportation" protocols which succeed or fail independently - the success probability of each is $n /(n+1)$. The KLM-type gate succeeds if the two $F$-teleportations succeed, thus the success probability of this (CPHASE) gate is $n^{2} /(n+1)^{2}$. We shall henceforth denote such gates as $C Z_{(n)}$. The conditional gate operation is achieved due to the entangled state $\left(\left|C S_{n}\right\rangle\right)$ of the $2 n$ ancillary photons. As defined in [1], $\left|C S_{n}\right\rangle=\sum_{i, j=0}^{n}(-1)^{(n-i)(n-j)}\left|t_{n}^{i}\right\rangle\left|t_{n}^{j}\right\rangle$, where $\left|t_{n}^{i}\right\rangle=$ $|1\rangle^{i}|0\rangle^{n-i}|0\rangle^{i}|1\rangle^{n-i}$ is the state of $2 n$ modes carrying exactly $n$ photons, that take part in one $F$-teleportation (in $\left|t_{n}^{i}\right\rangle$ unlike the rest of the paper, $\{|0\rangle,|1\rangle\}$ are states of zero and one photon in a mode). If the operation of $C Z_{(n)}$ succeeds then a negative phase is introduced to the state $|1\rangle_{p_{2}^{\prime}}|1\rangle_{q_{2}^{\prime}}$, and the state of the overall system becomes:

$$
|0 \downarrow\rangle_{p_{1} p_{2}}|0 \uparrow\rangle_{q_{1} q_{2}}(|00\rangle+|01\rangle+|10\rangle-|11\rangle)_{p_{2^{\prime} q_{2^{\prime}}}}+
$$




$$
\begin{gathered}
|0 \uparrow\rangle_{p_{1} p_{2}}|1 \leftrightarrow\rangle_{q_{1} q_{2}}(|00\rangle-|01\rangle+|10\rangle+|11\rangle)_{p_{2^{\prime}} q_{2^{\prime}}}+ \\
|1 \leftrightarrow\rangle_{p_{1} p_{2}}|0 \uparrow\rangle_{q_{1} q_{2}}(|00\rangle+|01\rangle-|10\rangle+|11\rangle)_{p_{2^{\prime}} q_{2^{\prime}}}+ \\
|1 \leftrightarrow\rangle_{p_{1} p_{2}}|1 \leftrightarrow\rangle_{q_{1} q_{2}}(|00\rangle-|01\rangle-|10\rangle-|11\rangle)_{p_{2^{\prime}} q_{2^{\prime}}}
\end{gathered}
$$

At this stage photons $p_{2^{\prime}}$ and $q_{2^{\prime}}$ are measured in the basis $\{|+\rangle,|-\rangle\}$ (the $x$-basis). Taking for example the first line in (3), the term within the brackets can be written as $(|++\rangle+|+-\rangle+|-+\rangle-|--\rangle)$. The terms within the brackets in the other lines correspond to similar expressions only with the minus sign appearing in different places. Therefore, upon receiving the outcome $|++\rangle$ in the measurement, the resulting state of photons $\left\{p_{1}, p_{2}\right\}$ and $\left\{q_{1}, q_{2}\right\}$ would be exactly as in Eq. (2), while other results would lead to similar states up to local phase adjustments. A failure in the operation of $C Z_{(n)}$ would result in the measurement in the $z$-basis of either $p_{2^{\prime}}$ or $q_{2^{\prime}}$. Taking $p_{2^{\prime}}$ for example, we can see from the initial state of the link that whether we get an outcome of $|0\rangle$ or $|1\rangle$, the path of $p_{1}$ will remain maximally entangled with the polarization of $p_{2}$, enabling the transmission of the data forward to the next link.

Let us address the construction of the chains. The basic units from which the chain is constructed are "twophoton units" consisting of a linked-photon $\left(p_{i}\right)$ and a free-arm photon $\left(p_{i}^{\prime}\right)$ in a maximally entangled state. Such two-photon units can be produced from four single (non-entangled) photons, by using for example the gate $c-z_{1 / 16}$ of KLM [1]. Thus, the basic requirements of our scheme are similar to those of the KLM-scheme. In each step in the construction an attempt is made to connect such a two-photon unit to the already constructed chain (Fig. 1). One can entangle, by using CPHASE KLM-type gates $-C Z_{(n)}$, the path degree of freedom of the last photon in the chain to the polarization of the linked-photon in the new unit. Since the polarization of the last photon in the chain is already entangled with the path of the previous photon, and this entanglement must be kept, two $C Z_{(n)}$ gates must be applied - one for each of the polarization modes of the photon [2]. The operation, therefore, includes four $F$-teleportations. Clearly, it is more efficient to apply first the two teleportations on the new pair, minimizing the risk of removing photons from the chain. Thus, for each random walk step we have an "off-line" preparation in which (the first) two $F$-teleportation steps are applied to a two-photon unit using two $\left|C S_{n}\right\rangle$ states. The resulting "prepared unit" will then be used when applying the two $F$-teleportation to the last photon in the chain.

The efficiency of the new method can be readily calculated since the construction of a chain is simply a one dimensional random walk process. A step forward is taken when the operation of connecting a new two- photon unit to the chain has succeeded. A step backwards is taken when this operation fails in such a way that the last linked-photon in the chain was measured (removing also a free-arm photon). Because of the fact that a separate $F$-teleportation is applied to each of the polarizations of the last photon in the chain, there is also a possibility to fail without measuring the last photon in the chain, leaving the chain unaffected (while destroying just the prepared-unit). In the random walk analysis only the two $F$-teleportations applied to the last photon in the chain are considered. Therefore the probability of success is $p=n^{2} /(n+1)^{2}$. The probability to fail destructively and remove a linked photon is $q=(1-p) / 2=(2 n+1) / 2(n+1)^{2}$ (see [2]). For a one dimensional random walk the average number of attempts required in order to advance one step forward (for a large number of steps) is simply $R(n)=1 /(p-q)$. In terms of $n$ - the parameter of the KLM-type gate we obtain $R(n)=2(n+1)^{2} /\left(2 n^{2}-2 n-1\right)$. In order to obtain the amount of resources, in terms of ancillary states, consumed in the process, we need to calculate the cost of a single random walk step. Since one applies two $F$-teleportations on the two-photon unit, before a similar operation is applied on the last photon in the chain, on average we will need $(n+1)^{2} / n^{2}$ attempts in order to succeed, each costing a single two-photon unit. In each of these attempts one $\left|C S_{n}\right\rangle$ (in case the first $F$ teleportation fails) or two such states (in all other cases) are consumed as well. On average in each random walk step - whether successful or not - $(n+1)^{2} / n^{2}$ two-photon units and $(2 n+1)(n+1) / n^{2}$ copies of $\left|C S_{n}\right\rangle$ are consumed. The resources required on average in order to add a two photon unit to the chain are given by multiplying the above expressions by $R(n)$ :

$$
\begin{aligned}
& \frac{2(n+1)^{4}}{n^{2}\left(2 n^{2}-2 n-1\right)} \text { 2-photon units } \\
& \frac{2(n+1)^{3}(2 n+1)}{n^{2}\left(2 n^{2}-2 n-1\right)} \text { copies of }\left|C S_{n}\right\rangle
\end{aligned}
$$

What is the average number of links, per two-qubit gate, that a chain must include? As each of the independent $F$-teleportations constituting a $C Z_{(m)}$ gate succeeds with probability of $m /(m+1)$, for each two-qubit gate a chain must have, on average, $(m+1) / m$ links with free arms. We can now summarize the efficiency analysis. On average, in order to implement a single two-qubit gate that is to construct the required length in two chains and connect them - the following resources are required (for a large number of gates):

$$
\begin{gathered}
\frac{2(m+1)}{m} \frac{2(n+1)^{3}(2 n+1)}{n^{2}\left(2 n^{2}-2 n-1\right)} \text { copies of }\left|C S_{n}\right\rangle \\
\frac{2(m+1)}{m} \frac{2(n+1)^{4}}{n^{2}\left(2 n^{2}-2 n-1\right)} \text { 2-photon units } \\
\frac{(m+1)^{2}}{m^{2}} \text { copies of }\left|C S_{m}\right\rangle
\end{gathered}
$$

where chain construction is based on $C Z_{(n)}$ gates and 


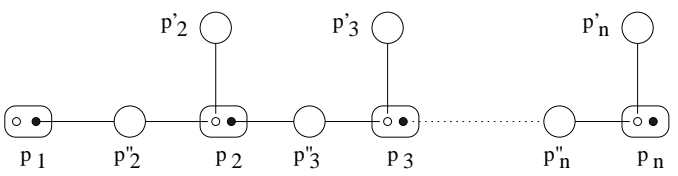

FIG. 2: A chain state for the linked-state model with inert photons (denoted by double primes) connected in between the linked-photons.

the connections between the chains are carried out using $C Z_{(m)}$ gates.

As can be easily verified, in the random walk process we obtain $p>q$ when using any $C Z_{(n)}$ with $n \geq 2$, which means that the simplest ancillary states with which a chain can be efficiently prepared are the 4-photon states $\left|C S_{2}\right\rangle$. (For comparison, in the original method 2] the simplest KLM-type gate, with which the linked-state can be constructed in a straightforward way, requires the state $\left|C S_{3}\right\rangle$.) The process of establishing a connection between two chains can use any KLM-type gate (namely, $C Z_{(m)}$ with $m \geq 1$ ), as it does not affect any part of the chains except the free arms.

In order to improve the efficiency of our method one can employ "inert photons" as was done in the original scheme [2]. These are additional photons included in the chain, that do not take part in the operation of the logic gates. Their only purpose is to improve the bias of the random walk process, by decreasing the risk of losing the last linked-photon in the chain. Constructing the chain in this way one adds in each step a 3-photon unit (as shown in Fig. 2) — the linked-photon with its free-arm and an inert photon. Clearly, each random walk step would now include more operations, however, at least for low values of $n$ the overall construction would be more efficient. Alternatively, one can improve the random walk bias of the basic free-arm method by adding to the chain a number of connected two-photon units in each step. By using each of these methods it is possible to construct the chain using the simplest KLM-type gate, $C Z_{(1)}$ []].

The "free-arm photon" method can be applied to the optical cluster state model as well. In this model each photon carries a single relevant two-dimensional degree of freedom and a chain is constructed by entangling each photon to its two nearest neighbors. As in the linkedstate model, here also the data is being processed while progressing along the chain. However this is done not through measurements in the Bell-basis but by measurements of single photons (i.e. a single degree of freedom). Due to the different structure of the chain single-qubit gates are not implemented directly but through singlephoton measurements as well.

We suggest now a scalable "free-arm optical cluster state model". The chain that we consider is shown in Fig. 3. Each third photon is connected to free-arm photon. After connecting it to another free-arm photon of

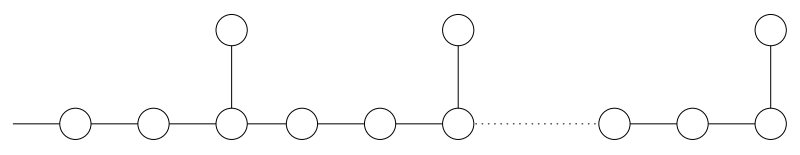

FIG. 3: A chain state for the cluster state model.

a different chain the resulting four-photon state can be used to implement a conditional phase flip gate. The photons without free-arms are required in order to apply a (general) single-qubit gate, between any two consecutive two-qubit gates. A simple way to construct the above chain is, for example, to add in each step a 4-photon unit as depicted in Fig. 3. If an attempt to add such a unit fails then one can still try and fix the chain (damaged because of this failure). Only a series of three gate failures would destroy the last 4-photon unit in the chain. The resources required for the addition of one 4-photon unit to the chain are $(n+1)^{4} /\left(n^{2}(n+1)^{2}-n\right)$ copies of 4-photon units and $(n+1)^{2}\left(n^{2}+3 n+3\right) /\left(n^{2}(n+1)^{2}-n\right)$ copies of $\left|C S_{n}\right\rangle$, with $n \geq 1$ (details are given in [7]).

The scalable models that we have presented above can be used to implement general-purpose quantum computation: instead of preparing the custom-made linked (or cluster) state which corresponds to the specific computation, it would be sufficient to construct standard-form chains. The weaving of the chains can then be carried out in conjunction with the evolution stage. Each connection between chains can be established just before the corresponding two-qubit gate is executed (that is, before the connected photons are measured). As the data carried by the chains is not affected by a failed connection attempt, one has simply to re-try to connect the chains using the next free-arm. Clearly, in this way, given that a long enough chain has been prepared for each qubit, any computation can be performed with the same efficiency as calculated above.

This work was supported in parts by the Israel Science Foundation - FIRST (grant \#4088103). N. Y. acknowledges the Aly Kaufman Fellowship, Technion, and the UK EPSRC (grant GR/527405/01).

[1] E. Knill, R. Laflamme, and G.j. Milburn, Nature 409, 46 (2001).

[2] N. Yoran and B. Reznik, Phys. Rev. Lett. 91, 037903 (2003).

[3] D. Boschi, S. Branca, F. De Martini, L. Hardy, and S. Popescu, Phys. Rev. Lett. 80, 1121 (1998).

[4] M. A. Nielsen, Phys. Rev. Lett. 93, 040503 (2004).

[5] R. Raussendorf and H. J. Briegel, Phys. Rev. Lett. 86, 5188 (2001).

[6] D. E. Browne and T. Rudolph, Phys. Rev. Lett. 95, 010501 (2005)

[7] T. Mor and N. Yoran, In preparation. 